\title{
Prediction of Remaining Useful Life of Wind Turbine Shaft Bearings Using Machine Learning
}

Jinsiang Shaw

Institute of Mechatronic Engineering, National Taipei University of Technology, Taipei, Taiwan, jshaw@ntut.edu.tw

Bingjie Wu

Institute of Mechatronic Engineering, National Taipei University of Technology, Taipei, Taiwan

Follow this and additional works at: https://jmstt.ntou.edu.tw/journal

Part of the Fresh Water Studies Commons, Marine Biology Commons, Ocean Engineering Commons, Oceanography Commons, and the Other Oceanography and Atmospheric Sciences and Meteorology Commons

\section{Recommended Citation}

Shaw, Jinsiang and Wu, Bingjie (2021) "Prediction of Remaining Useful Life of Wind Turbine Shaft Bearings Using Machine Learning," Journal of Marine Science and Technology. Vol. 29: Iss. 5, Article 4.

DOI: $10.51400 / 2709-6998.2465$

Available at: https://jmstt.ntou.edu.tw/journal/vol29/iss5/4

This Research Article is brought to you for free and open access by Journal of Marine Science and Technology. It has been accepted for inclusion in Journal of Marine Science and Technology by an authorized editor of Journal of Marine Science and Technology. 


\title{
Prediction of Remaining Useful Life of Wind Turbine Shaft Bearings Using Machine Learning
}

\author{
Jinsiang Shaw ${ }^{\mathrm{a}, \mathrm{b}, *}$, Bingjie $\mathrm{Wu}{ }^{\mathrm{a}}$ \\ ${ }^{a}$ Institute of Mechatronic Engineering, National Taipei University of Technology, Taipei, Taiwan \\ ${ }^{\mathrm{b}}$ Research Center of Energy Conservation for New Generation of Residential, Commercial, and Industrial Sectors, National Taipei \\ University of Technology, Taipei, Taiwan
}

\begin{abstract}
Wind turbines are a major trend in the current green energy market. Wind energy is abundant, and if utilized properly, can result in significant reductions in carbon emissions. Therefore, the development of wind power systems is urgently required. However, wind turbines are mainly built in unmanned areas. Regular inspections require substantial manpower and material resources, and doubts regarding the accuracy of the inspected data may occur. Therefore, it is necessary to establish an automatic diagnostic method for determining the remaining useful life (RUL) of a wind turbine to facilitate predictive maintenance. In this study, a multi-class support vector machine (SVM) and a convolutional neural network (CNN) were employed for fault diagnosis and RUL prediction of the shaft bearings used in wind turbines. During the multi-SVM process, the vibration signal of the shaft bearings was converted into a 15-parameter feature vector input for training and prediction; we achieved a resulting classification accuracy of $95.33 \%$. For the CNN process, the spectrogram of the vibration signal from the wind turbine shaft bearings was used to train a CNN; here, we achieved a resulting classification accuracy of $100 \%$.
\end{abstract}

Keywords: CNN, SVM, RUL, Machine learning

\section{Introduction}

$\mathrm{E}$ nvironmental awareness, including carbon emission restrictions, has increased in recent years. The demand for electricity has increased with industrial developments and additional power plants are required to provide stable electricity. To address the environmental impact, policies for regulating air pollution and the use of nuclear power have been implemented. In addition, countries are actively considering alternative energy sources such as geothermal, hydraulic, tidal, solar, and wind. Wind power has garnered significant attention and is an important source of global energy. Wind turbines are built on stable wind farms and generally on unmanned land. In addition to the difficulty of construction, equipment maintenance is a challenge. The repair of failed equipment is time consuming and a considerable amount of financial resources is spent on restoring power generation. Therefore, it is extremely important to be aware if a fault occurs in advance. Such faults were detected manually in the early days. Recently, intelligent fault diagnosis [11] and predictive fault diagnosis [23]) have garnered significant attention and can reduce the manpower required to evaluate and provide timely forecasts of impending malfunctions. An increasing amount of research has been conducted on the damage prediction of rotating machinery, including bearings and gears. In early machine learning, expert systems [6], artificial neural networks (ANNs) $[1,16,20]$ and support vector machines (SVMs) $[9,19,29,31]$ were used for fault diagnosis. However, it is necessary to manually extract the characteristics of the data, such as time,

Received 30 July 2020; revised 30 September 2020; accepted 27 May 2021.

Available online 18 November 2021.

* Corresponding author. Institute of Mechatronic Engineering, National Taipei University of Technology, Taipei, Taiwan.

E-mail address: jshaw@ntut.edu.tw (J. Shaw). 
frequency, or time-frequency domain features $[10,12]$. These characteristics indicate the health of the machine. Therefore, they are used as inputs to the diagnostic model to predict whether the machine will continue to operate normally. Recently, deep learning diagnostic models such as stacked autoencoders [5,13,15], convolutional neural networks (CNNs) [28,30], and ResNet [17] have been employed. Deep learning models can automatically extract data characteristics, thereby significantly reducing human labor. In addition, providing additional training data to the deep learning model can improve model accuracy.

This study mainly uses a multi-SVM and deep learning CNN model to develop a health status diagnosis model. The vibration signals of the damaged shaft bearings of a wind turbine with a 2 MW power output were collected. The data were sampled at $97,656 \mathrm{~Hz}$ for $6 \mathrm{~s}$ per day. A total of fifty days of data were collected when the shaft bearings failed on the last day [3]. The objective of our study is to predict the remaining useful life (RUL) of the shaft bearings of a wind turbine; for example, five days of RUL before failure, given the collected vibration signals of a particular day (day 45). Lin [14] applied a probabilistic neural network (PNN) to predict the RUL of shaft bearings with an accuracy of only $23 \%$. By adding the sideband energy ratio (SER) and sideband power factor (SBPF) in the feature vector, the classification accuracy attained was $93.6 \%$. In this study, we employed a multi-SVM and deep learning CNN model to predict the health status of the shaft bearings to enable easy deduction of the RUL of the bearings.

\section{Prediction model}

\subsection{Support vector machine}

SVM is a binary classifier. An optimal hyperplane capable of accurately predicting the data was investigated for the training data projected into a high-dimensional feature space. Vapnik and Chervonenkis [22] developed the first SVM model. Later, Drucker et al. [4] published an SVM based on the statistical learning theory; the SVM used only a small dataset to produce a reliable prediction. In addition, SVMs have been used by several scholars to successfully classify faults in various mechanical parts [26,27].

Furthermore, a multi-SVM, which is not limited to dichotomy but can carry out multiple classifications, has been proposed [25]. There are two methods for applying SVMs to multiple classifications. The first is the one-vs-rest method. To sort the results, the corresponding $n$ SVMs are established. The data to be classified are fed into $n$ SVMs as the input. Finally, the maximum value of the $n$ SVM outputs is used to determine the classification to which the data belongs.

The second method is the one-vs-one method. From $n$ classes of data, any two classes of data must be chosen to conduct SVM training. This action is repeated until all categories have corresponding SVMs. Therefore, $n(n-1) / 2$ SVMs are generated. Finally, we feed the data to be classified into trained models. The category with the highest score is subsequently determined. In this study, we employ the one-vs-one method for classifying the input signal of shaft bearings corresponding to which day it represents, namely from day 1 up to day 50 .

\subsection{Convolutional neural network}

CNNs have two main layers: convolution and pooling layers. A convolutional layer composed of $3 \times 3,5 \times 5$, or $7 \times 7$ filters is an important layer for feature extraction. A convolution operation is applied to the input image using a mask to obtain the feature map of the image. The pooling layer is a powerful tool used by a CNN to compress images while retaining important information. The pooling layer can select a different window size and typically maximum pooling is employed.

CNNs have gained importance in recent years and have achieved significant performance in image recognition. CNN debuted in 2012 at the ImageNet Large Scale Visual Recognition Challenge (ILSVRC), where the championship model was AlexNet. Since then, NfNet, VggNet, GoogleNet, and Microsoft ResNet network models have been applied to image classification [8]. GoogleNet, used in this study, was the champion model at the ILSVRC in 2014. It is connected by a large number of inception structures, which can reduce the model parameters and enrich the diversity of the learning characteristics. A total of 27 layers of GoogleNet model [21] were compared with a 122-layer ResNet model [7]; it was found that the training time of GoogleNet was faster than that of ResNet.

\section{Feature extraction}

Data from a 6-s vibration signal of a high-speed shaft bearing of a $2 \mathrm{MW}$ wind turbine were taken as the training data each day $(585,936)$ for a total of fifty days, as shown in Fig. 1. It is visible that the trend of the vibration amplitude increases with time (day); however, the amplitude does not monotonically increase and exhibits a deteriorating condition until 


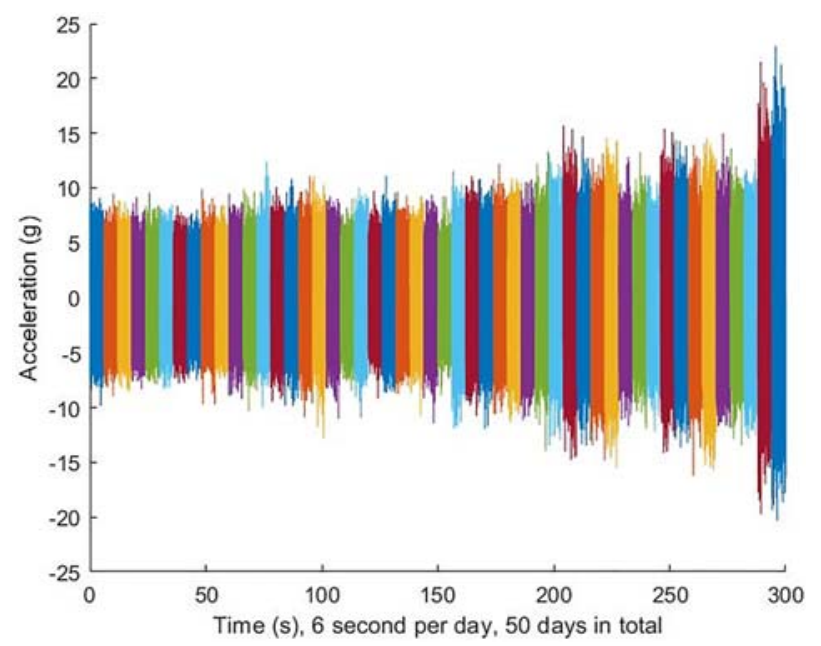

Fig. 1. Vibration signal in the time domain.

the last day of breakdown. The root-mean-square (RMS) amplitude per day is illustrated in Fig. 2, where the RMS amplitude and time (day) are not one-to-one.

\subsection{Vibration-related characteristics}

During the operation of a wind turbine, the rotational speed is slightly time-varying and not in a stable state. The measured vibration signals include several characteristics. The fault signal is of an extremely small amplitude during an early mechanical part malfunction and is easily masked by surrounding noise. Therefore, it is important to obtain the fault signal in the initial stages. The characteristics of the vibration signal were extracted in both time and frequency domains. Each day, the measured vibration signal was sampled randomly into ten groups, each with 400,000 data points out of

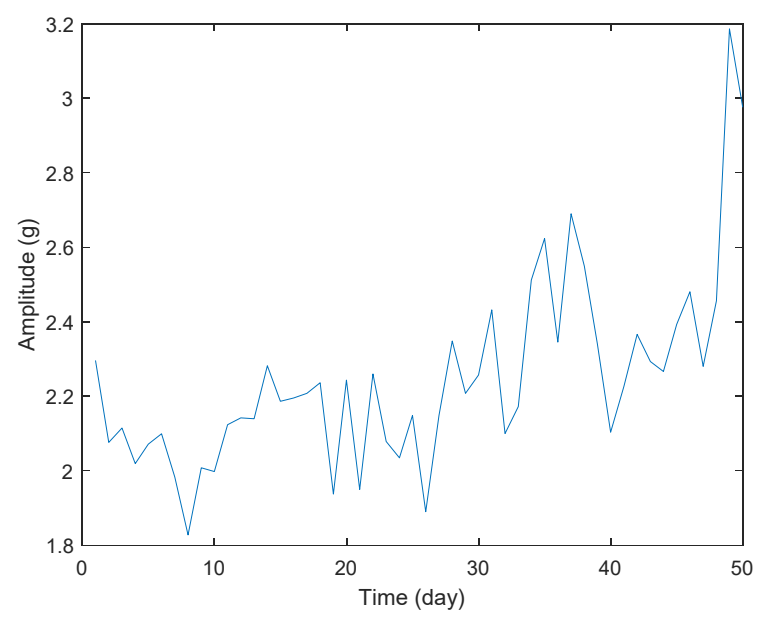

Fig. 2. RMS amplitude of vibration signal.
585,936 . The corresponding time and frequencydomain features for each group were then extracted. A total of fifteen feature values (eleven and four in the time and frequency domain, respectively) were obtained for each group and were fed into a multiSVM for training and prediction. The fifteen feature values that were chosen have been proved effective in indicating complex bearing faults and are independent of shaft speeds and loads $[14,18,24]$.

\subsubsection{Time domain}

All original data were in the time domain. Statistical features in the time domain can be used as the basis for identifying the health index. The eleven chosen feature values are listed in Table 1, where $x$ is the sampled vibration signal and $N$ is the number of sampled data points.

\subsubsection{Frequency domain}

In addition to the time-domain vibration features listed in Table 1, it is extremely important to include the frequency-domain features to improve the effectiveness of fault detection. In this study, features related to the spectral kurtosis (SK) of the vibration signal $x$ were added. The value of $\mathrm{SK}(\mathrm{f})$, defined in Eqn. (1), increases with the intensity of the fluctuations in the impulse amplitudes. Consequently, the value of SK can be used to indicate the severity of the damage. The SK of a signal $x(\mathrm{t})$ is defined as the kurtosis of its spectral components

Table 1. Statistical time-domain features.

\begin{tabular}{ll}
\hline Feature & Expression \\
\hline Mean & $\mu=\frac{1}{N} \sum_{i=1}^{N} x_{i}$ \\
Standard Deviation & $\sigma=\sqrt{\frac{1}{N-1} \sum_{i=1}^{N}\left|x_{i}-\mu\right|^{2}}$ \\
Root Mean Square & $\mathrm{RMS}=\sqrt{\frac{1}{N} \sum_{i=1}^{N}\left|x_{i}\right|^{2}}$ \\
Skewness & $\mathrm{E}\left[\frac{(x-\mu)^{3}}{\sigma^{3}}\right]$ \\
Kurtosis & $\mathrm{E}\left[\frac{(x-\mu)^{4}}{\sigma^{4}}\right]$ \\
Energy & $\frac{N}{i=1} x^{2}$ \\
Peak to Peak & $x_{\text {Max }}-x_{\text {Min }}$ \\
Crest Factor & $\frac{x_{M a x}}{R M S}$ \\
Shape Factor & $\frac{R M S}{\operatorname{Mean}(|x|)}$ \\
Impulse Factor & $\frac{x_{\text {Max }}}{\operatorname{Mean}(|x|)}$ \\
Margin Factor & $\frac{x_{\text {Max }}}{\operatorname{Mean}(|x|)^{2}}$ \\
\hline
\end{tabular}


and can be defined as a normalized fourth-order spectral moment as:

$\mathrm{SK}(\mathrm{f})=\frac{\left\langle\left|X^{4}(t, f)\right|\right\rangle}{\left\langle\left|X^{2}(t, f)\right|\right\rangle^{2}}-2$,

where $\langle\cdot\rangle$ represents the time-frequency averaging operator, $X^{4}(t, f)$ and $X^{2}(t, f)$ are the fourth- and second-order cumulants, respectively, of a bandpass filtered signal $x(\mathrm{t})$ around $f[2,18]$.

Once SK(f) is obtained, its skewness, kurtosis, average, and standard deviation can be calculated and included in the feature vector to predict the degradation of the wind turbine shaft bearings.

\subsection{Spectrum plot}

As shown in Fig. 2, the root-mean-square (RMS) amplitude per day cannot be used alone to determine the health status of the bearings owing to nonmonotonicity. Here, a fast Fourier transform (FFT) is used to obtain the frequency content of the vibration signal. For each day, a total of thirty spectrograms were generated, each with randomly chosen 500,000 data points for the FFT. A few samples of the resulting spectrograms are shown in Fig. 3. In the subsequent section, a trained $\mathrm{CNN}$ is employed to predict the day that a particular spectrogram represents.

\section{Experimental results}

\subsection{Support vector machine}

The multi-SVM input data is a feature vector. Feature extraction was performed to obtain fifteen feature values for each sampled time signal, as mentioned in Section 3.1. Seven out of the ten groups on each day were used for multi-SVM training. After completion of training, the remaining three groups were tested each day. The classification accuracy achieved was $95.33 \%(143 / 150)$.

The confusion matrix is listed in Table 2, where the recall rates of days 3 and 4 of the trained multiSVM are both zero. The precision rate for day 5 was only $33.33 \%(3 / 9)$ because the health conditions on days 3 and 4 were all misjudged as those of day 5; this mainly accounts for the loss of accuracy. On day 36 , the recall rate was only $66.67 \%(2 / 3)$ because one health condition of day 36 was misjudged to that of day 44 . The precision rate on day 44 was $75 \%(3 / 4)$. Note that only seven errors existed in the multiSVM predictions: three health conditions each of day 3 and day 4 misclassified as that of day 5; one day 36 health condition misclassified to that of day
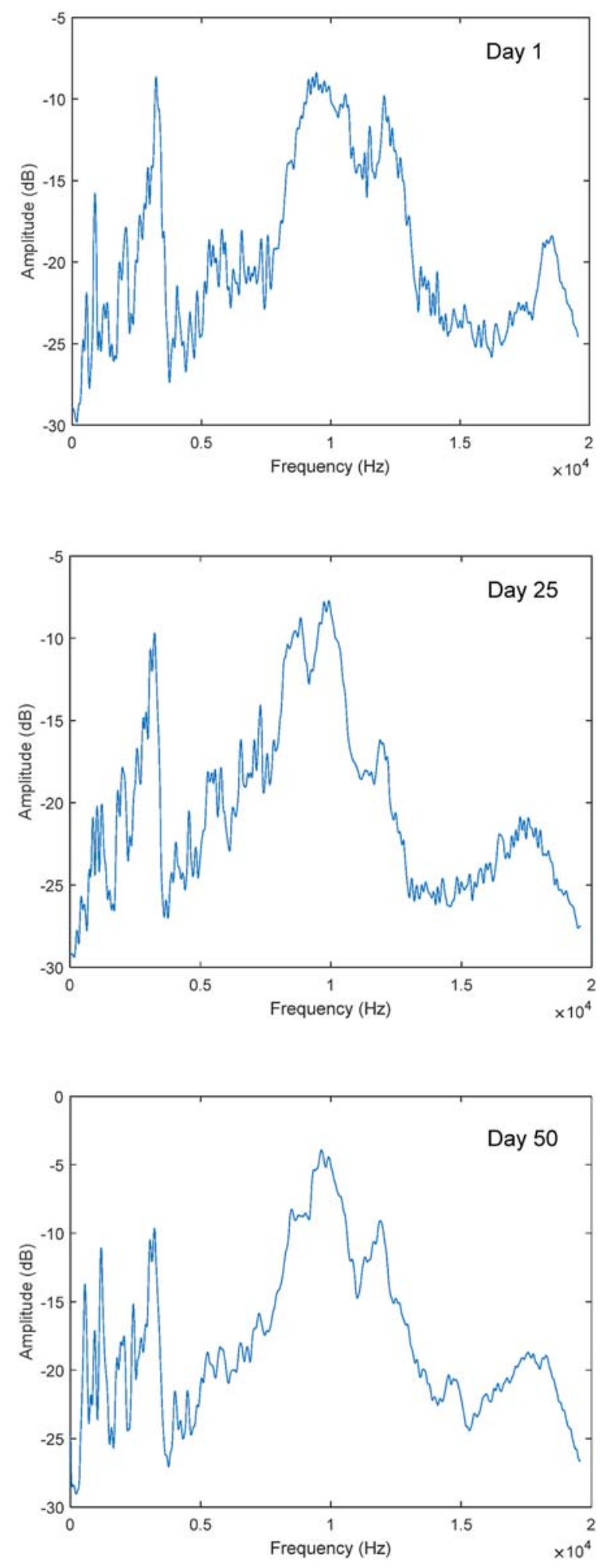

Fig. 3. Spectrogram of vibration signals.

44. The predicted RUL values of these seven cases were shorter than the true RUL values, which is acceptable in terms of predictive maintenance. It would be undesirable if the health condition of a 
Table 2. Confusion matrix of multi-SVM.

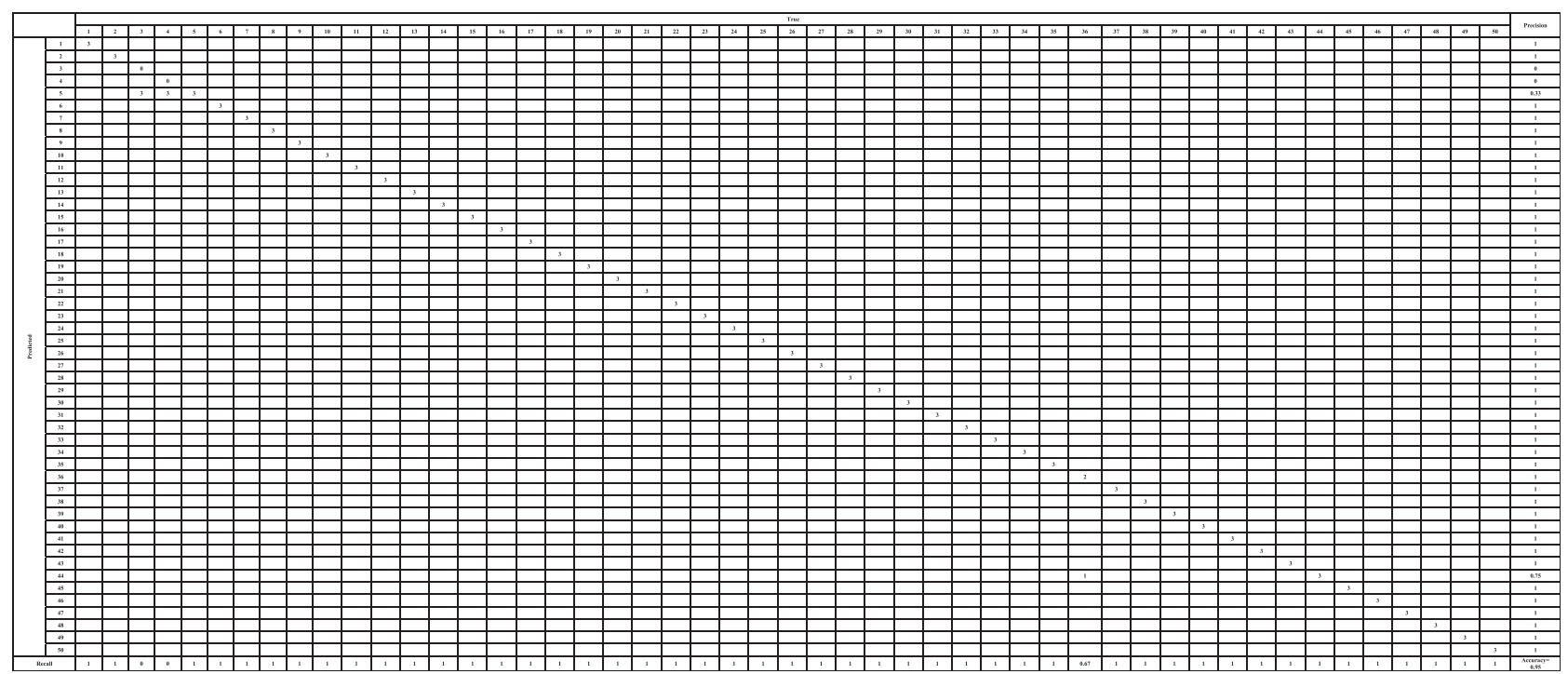

certain day is misjudged as that of an earlier day, which is more likely to cause maintenance problems. However, this does not occur in proposed multi-SVM model.

\subsection{Convolutional neural network}

2D images of the spectrogram, such as those displayed in Fig. 3, were used as the input for the CNN model to predict the health status that it would correspond to (with corresponding output labels as Day 1, Day 25, and Day 50, respectively). Thirty images per day were collected, resulting in a total of 1500 images as the dataset for the CNN. The GoogleNet model and transfer learning were employed here for the fifty days of classification. $70 \%$ of the images (1050 images) were used for training, and the remaining $30 \%$ (450 images) were used for

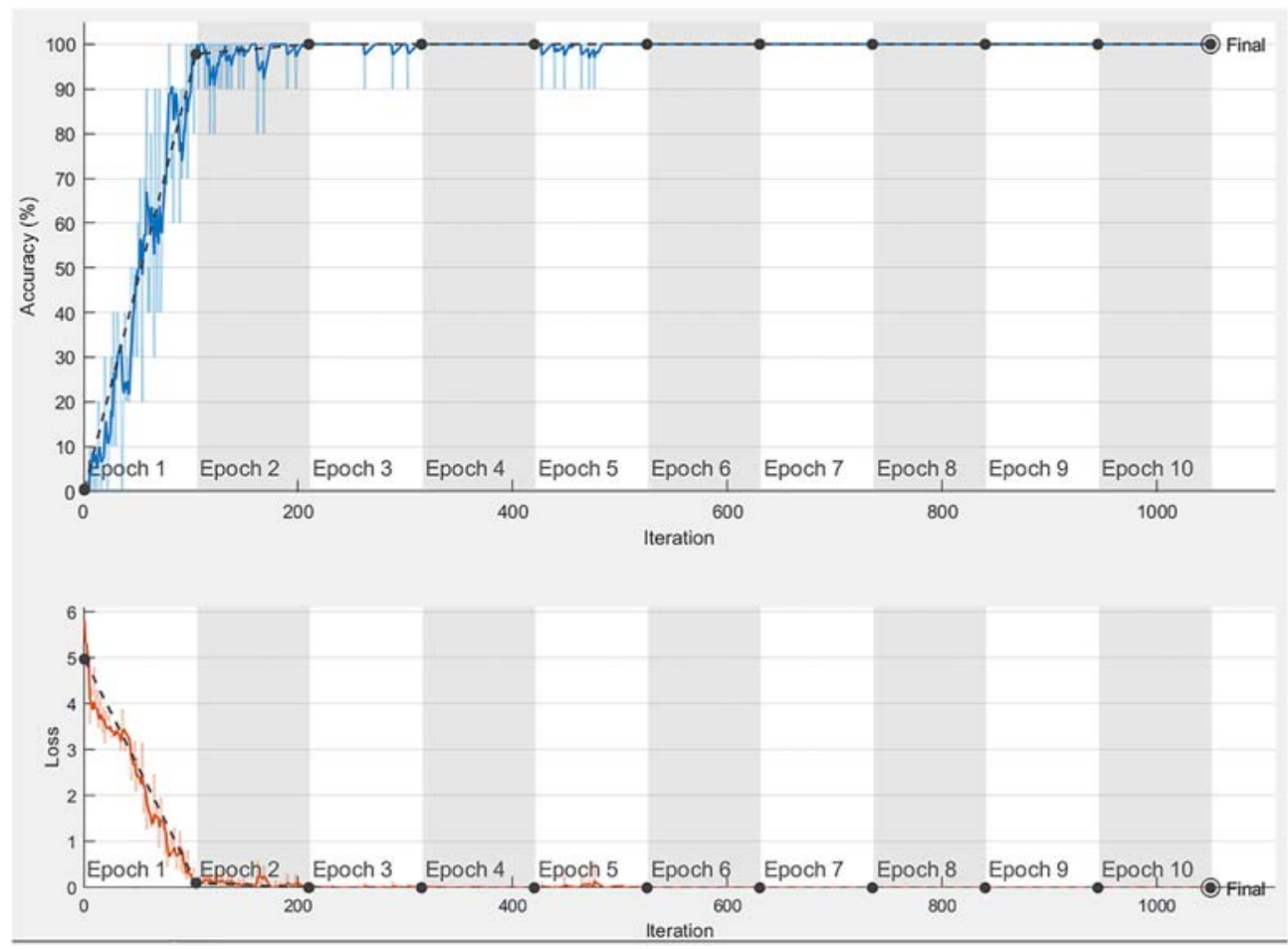

Fig. 4. CNN training. 
testing. The training process run in the MATLAB package is shown in Fig. $4 ; 100 \%$ accuracy was obtained for the training data. Finally, the accuracy achieved of the testing images of the trained GoogleNet model was $100 \%$. In other words, by transforming the measured vibration signal of any day to a spectrogram using FFT, the GoogleNet model can correctly identify the health condition of that specific day.

\section{Conclusion}

Both multi-SVM and GoogleNet CNN models were used to successfully predict the health condition of wind turbine shaft bearings in terms of the number of days for RUL. For the SVM model, an accuracy of $95.33 \%$ was obtained from the classification results. This result is superior to that achieved by Lin [14], who used a PNN model and achieved an accuracy of $93.6 \%$. CNN classifiers only need to transform the measured time series data into a spectrogram using FFT to classify the RUL with $100 \%$ accuracy; whereas SVM and PNN models require additional data preprocessing to extract time and frequency characteristics. Hence, it is advantageous to use CNN. Moreover, compared to PNN and SVM, CNN models are capable of achieving improved accuracy and reliability for wind turbine health identification. The scope of our future study involves the application of a long shortterm memory network (LSTM) to predict the RUL of the shaft bearing by directly using the sampled time series data as the input feature vector.

\section{Acknowledgment}

This work was financially supported by the "Research Center of Energy Conservation for New Generation of Residential, Commercial, and Industrial Sectors" from the Featured Areas Research Center Program within the framework of the Higher Education Sprout Project by the Ministry of Education (MOE) in Taiwan.

\section{References}

[1] Ahmed H, Nandi AK. Compressive sampling and feature ranking framework for bearing fault classification with vibration signals. IEEE Access 2018:44731-46.

[2] Antoni J. The spectral kurtosis: a useful tool for characterizing non-stationary signals. Mech Syst Signal Process 2006; 20(2):282-307.

[3] Bechhoefer E, Hecke BV, He D. Processing for improved spectral analysis. Ann Conf Prog Health Manage Soc 2013; $4(6)$.

[4] Drucker H, Wu D, Vapnik VN. Support vector machines for spam categorization. IEEE Trans Neural Network 1999;10(5): 1048-54.
[5] Duong BP, Kim JM. Non-mutually exclusive deep neural network classifier for combined modes of bearing fault diagnosis. Sensors 2018;18(4):1129. 2018.

[6] Ebersbach S, Peng Z. Expert system development for vibration analysis in machine condition monitoring. Expert Sys Appl 2008;34(1):291-9.

[7] He K, Zhang X, Ren S, Sun J. Deep residual learning for image recognition. 2015

[8] İnik Ö, Ceyhan A, Balcõoğlu E, Ülker E. A new method for automatic counting of ovarian follicles on whole slide histological images based on convolutional neural network. Comput Biol Med 2019;112. Article ID 103350.

[9] Islam MMM, Kim JM. Reliable multiple combined fault diagnosis of bearings using heterogeneous feature models and multiclass support vector machines. Reliab Eng Syst Saf 2019;184:55-66.

[10] Lei Y, He Z, Zi Y, Hu Q. Fault diagnosis of rotating machinery based on multiple ANFIS combination with gas. Mech Syst Signal Process 2007;21(5):2280-94.

[11] Lei Y, Yang B, Jiang X, Feng J, Li NP, Asoke KN. Applications of machine learning to machine fault diagnosis: a review and roadmap. Mech Syst Signal Process 2020;138. Article ID 106587.

[12] Lei Y, Zuo MJ, He Z, Zi Y. A multidimensional hybrid intelligent method for gear fault diagnosis. Expert Syst Appl 2010;37(2):1419-30.

[13] Li C, Zhang W, Peng G, Liu S. Bearing fault diagnosis using fully-connected winner-take-all autoencoder. IEEE Access 2018;6:6103-15.

[14] Lin L. Fault diagnosis and remaining useful life prediction of the high-speed shaft gear and bearing in wind turbine drivetrain. Master Thesis. Taiwan: Department of Mechanical and Mechatronic Engineering, National Taiwan Ocean University; 2019 (in Chinese).

[15] Liu H, Li L, Ma J. Rolling bearing fault diagnosis based on STFT-deep learning and sound signals. Shock Vib 2016;2016. Article ID 6127479.

[16] Muruganatham B, Sanjith MA, Krishnakumar B, Murty S. Roller element bearing fault diagnosis using singular spectrum analysis. Mech Syst Signal Process 2013;35(1-2): 150-66.

[17] Peng D, Liu Z, Wang H, Qin Y, Jia L. A novel deeper onedimensional $\mathrm{CNN}$ with residual learning for fault diagnosis of wheelset bearings in high-speed trains. IEEE Access 2019; 7:10278-93.

[18] Saidi L, Ali JB, Bechhoefer E, Benbouzid M. Wind turbine high-speed shaft bearings health prognosis through a spectral Kurtosis-derived indices and SVR. Appl Acoust 2017;120: 1-8.

[19] Saidi L, Ali JB, Friaiech F. Application of higher order spectral features and support vector machines for bearing faults classification. ISA (Instrum Soc Am) Trans 2015;54:193-206.

[20] Samanta B, Al-Balushi KR. Artificial neural network based fault diagnostics of rolling element bearings using timedomain characteristics. Mech Syst Signal Process 2003;17(2): 317-28.

[21] Szegedy C, Liu W, Jia Y, Sermanet P, Reed S, Anguelov D, Erhan D, Vanhoucke V, Rabinovich A. Going deeper with convolutions. Proc IEEE Conf Comput Vis Pattern Recognit 2015:1-9.

[22] Vapnik VN, Chervonenkis AY. On the uniform convergence of relative frequencies of events to their probabilities. Theor Probab Appl 1971;16(2):264-80.

[23] Wang J, Liang Y, Zheng Y, Gao RX, Zhang F. An integrated fault diagnosis and prognosis approach for predictive maintenance of wind turbine bearing with limited samples. Renew Energy 2020;145:642-50.

[24] Wang X, Zheng Y, Zhao Z, Wang J. Bearing fault diagnosis based on statistical locally linear embedding. Sensors (Basel) 2015;15(7):16225-47.

[25] Weston J, Watkins C. Support vector machines for multiclass pattern recognition. ESANN; 1999. 
[26] Yin Z, Hou J. Recent advances on SVM based fault diagnosis and process monitoring in complicated industrial processes. Neurocomputing 2016;174(Part B):643-50.

[27] Yuan S, Chu F. Support vector machines-based fault diagnosis for turbo-pump rotor. Mech Syst Signal Process 2006; 20(4):939-52.

[28] Zhao D, Wang T, Chu F. Deep convolutional neural network based planet bearing fault classification. Comput Ind 2019; 107:59-66.

[29] Zhu K, Li H. A rolling element bearing fault diagnosis approach based on hierarchical fuzzy entropy and support vector machine. Proc IME C J Mech Eng Sci 2016;230(13): 2314-22.

[30] Zhu Z, Peng G, Chen Y, Gao H. A convolutional neural network based on a capsule network with strong generalization for bearing fault diagnosis. Neurocomputing 2019; 3235:62-75.

[31] Ziani R, Felkaoui A, Zegadi R. Bearing fault diagnosis using multiclass support vector machines with binary particle swarm optimization and regularized Fisher's criterion. J Intell Manuf 2017:1-13. 